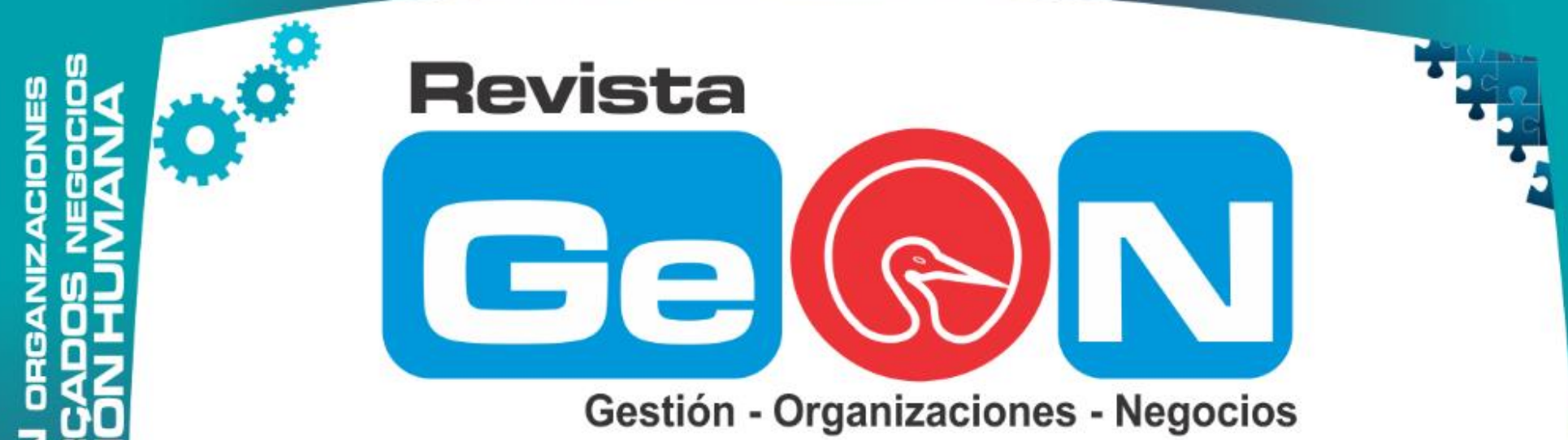

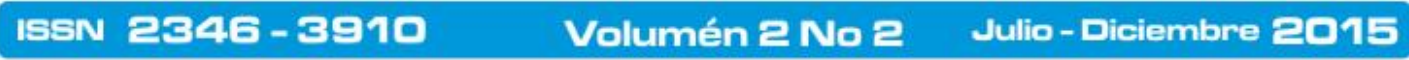

Revista Electrónica de la Facultad de Ciencias Económicas de la Universidad de los Llanos

1. 3 II

440

VILLAVICENCIO - COLOMBIA

$>2$

64

2

2 平品

70

ব)

展

o

ii III

0 U

ใด

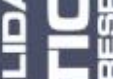

居的

임

Iiin in

照

只

造

iil 2

if in

4 吕

2

400

付 iI
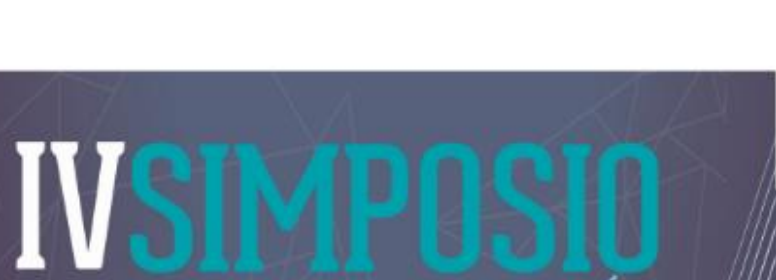

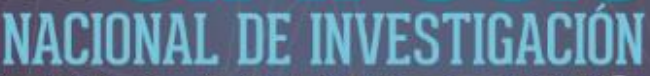

EN MARKETING
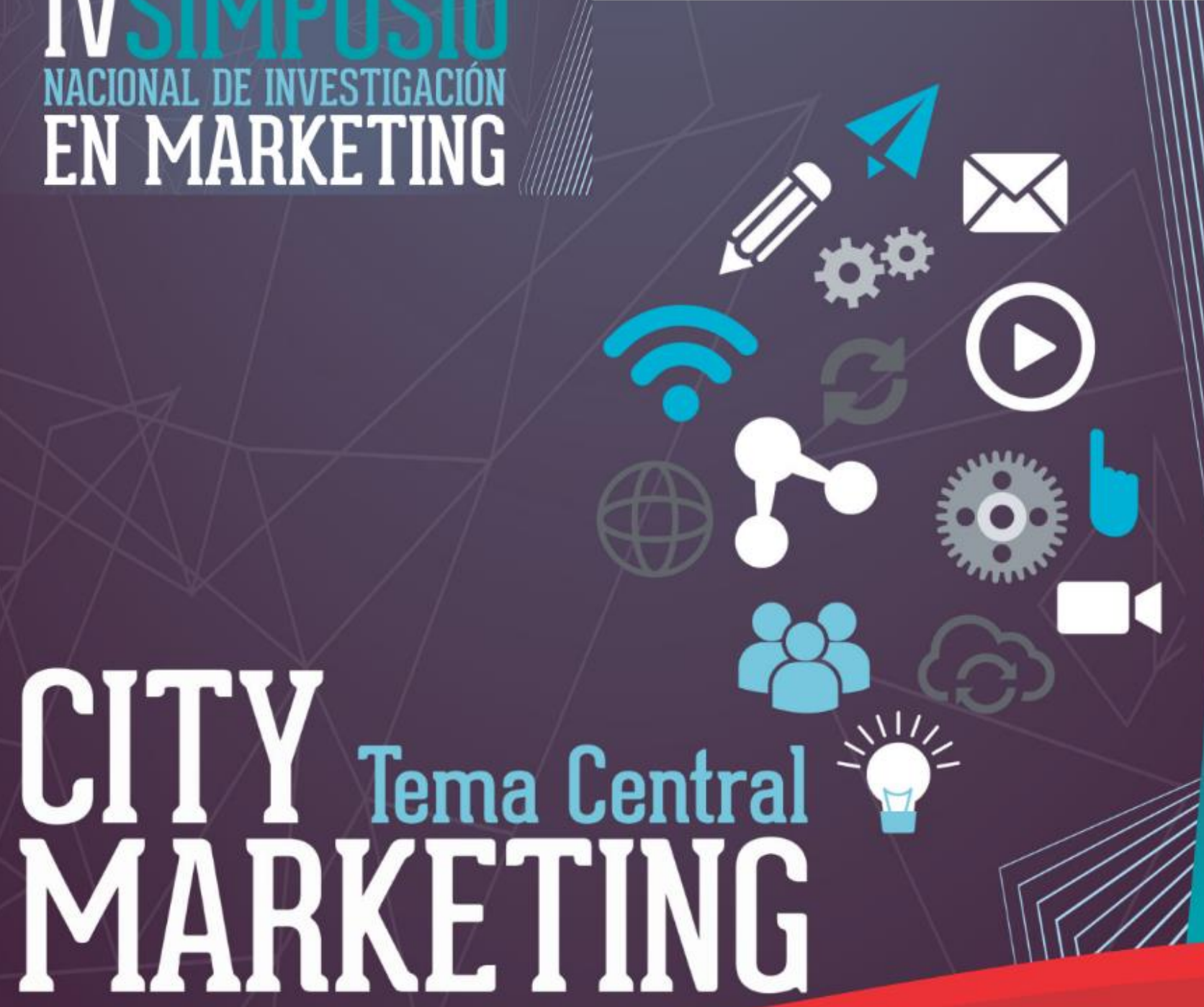

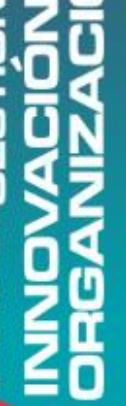
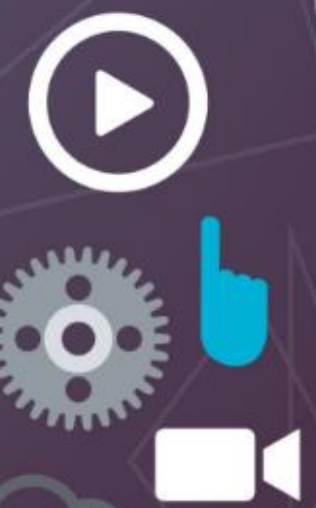


\section{Sexshop: Tendencias y percepciones entre jóvenes universitarios - Daniel Ruiz - Madeline Melchor}

Daniel Alejandro Ruiz Alzate.

Estudiante de Comunicación Publicitaria. Universidad Autónoma de Occidente daaruizal@gmail.com

Madeline Melchor Cardona:

Magister en Ingeniería.

mmelchor@uao.edu.co

\section{Resumen}

El concepto sobre sexualidad ha cambiado con el tiempo y actualmente se observan nuevos comportamientos enfocados hacia la diversidad, que se pueden reflejar por ejemplo en la apertura de tiendas Sexshop que comercializan juguetes sexuales. La presente investigación explora las percepciones y actitudes cognitivas, respecto a las prácticas asociadas al comportamiento de compra en tiendas Sexshop entre jóvenes universitarios. Se trabajó a través de un enfoque mixto en dos etapas Cualitativa (grupo focal) Cuantitativa (cuestionario) encontrando que gran parte del segmento en estudio encuentra cómodo hablar sobre sexualidad, existe una percepción mayor de uso de juguetes sexuales por parte de las mujeres y que la publicidad en el negocio debe ser reconsiderada por su poco impacto.

\section{Palabras clave}

Tiendas; juguetes sexuales; comportamiento del consumidor.

\footnotetext{
Abstract

The concept of sexuality has changed over time and new behaviors focused on diversity, which can be reflected for example in opening shops sex shops that sell sex toys. This research explores the cognitive perceptions and attitudes regarding practices related to buying behavior among university students Sexshop shops. We worked through a mixed approach in two
}

Qualitative stages (focus group) - Quantitative (questionnaire) finding that much of the segment under study are comfortable talking about sexuality, there is a greater perception of sex toy use by women and advertising in business should be reconsidered its little impact.

\section{Keywords}

Sexshop; Sex toys; Perception.

\section{Introducción}

Los sex-shop o tiendas de artículos sexuales pertenecen a un mercado que viene creciendo y haciendo cada vez más visible, dado que se manejaba de forma oculta y cubierta por los estigmas que cargaba con relación a las personas usuarias de estos productos. (Caraballo, 2010; Ramírez, 2011; Tabares, 2011)

Con relación al uso de juguetes sexuales se hace una diferenciación entre países europeos, Estados Unidos y Canadá, quienes avanzan de forma acelerada con respecto a Latinoamérica; esto posiblemente por su fuerte apego religioso, diferencia en clases sociales y menor acceso a la información (Mora, 2010). Esta diferencia se verá reflejada en las investigaciones previas relacionadas al tema, pues autores que trabajan desde Estados Unidos o Canadá encuentran un porcentaje alto de mujeres que usan juguetes sexuales solas o en compañía de su pareja, a la vez que se habla de esto como un tema natural que no representa un gran tabú, mientras que estudios realizados en Chile y Perú muestran una realidad distinta, donde son pocos los que hacen uso de estos juguetes sexuales, con un porcentaje representativo de personas que dijeron no sentirse atraídos por ellos.

De la mano con este crecimiento sostenible de la industria de juguetes sexuales y en general del sexo y los sexshop, en los últimos años ha explotado una bomba con nombre propio: "50 
shades of Grey". La autora del libro E.L. James ha creado su propia línea oficial de juguetes sexuales de la saga, cuyos kits son vendidos por toda Europa y ya ha vendido 130 mil artículos en tan solo tres meses; en Madrid ha habido un incremento del $30 \%$ en las ventas totales de juguetes eróticos (Finanzas Personales, 2015).

En Colombia, al igual que en el resto de países latinoamericanos podemos encontrar un crecimiento más lento del mercado, sin embargo este es cada vez más elevado, especialmente ahora que también ha sido alcanzado por el impacto de 50 shades of Grey. Nancy López, encargada de un sexshop dice que el $80 \%$ de las mujeres se acercan a buscar productos relacionados con la sumisión, específicamente artículos afines al libro. A Colombia aún no llegan los juguetes sexuales, los cuales tendrían un costo de 580000 COP, frente a 244000 COP aproximadamente de juguetes no oficiales de la saga (Finanzas Personales, 2015). Aunque no existen cifras oficiales del sector, se calcula que las tiendas sex shop en Colombia mueven alrededor de 1 millón de dólares al año ( Ramirez, 2011).

En cuanto al nivel regional, en el Valle del Cauca o específicamente en Cali aunque es fácil ver este tipo de locales, no se encuentra al mismo nivel de ciudades como en Bogotá, donde se pueden encontrar sexshop en prestigiosos centros comerciales los cuales se consideran de lujo. En Cali los locales siguen siendo tradicionales, en ubicaciones próximas entre ellos mismos y alejados de los centros comerciales.

Las principales zonas donde se pueden encontrar los sexshop en la ciudad de Cali, es en la Avenida sexta, como por ejemplo Kondomanía, la Avenida novena, la octava norte, unas cuantas en el sur, como My Love ubicada junto al San Andresito del sur y otras como la Pantera Roja que tiene distintos locales en toda la ciudad. De esta forma se puede decir que la mayor parte del mercado sexual en Cali se encuentra ubicado hacia el norte de la ciudad, sin descartar unos cuantos en el sur, que así mismo tienden a ser mejor organizados y más costosos, pues se dirigen a clases sociales más altas.

La presente investigación establece como objetivo identificar el comportamiento de compra y uso de los juguetes sexuales en el segmento de jóvenes universitarios, con el fin de conocer su percepción, preconcepciones $\mathrm{y}$ tendencias alrededor de los sex-shop. La información es de utilidad para las empresas del sector, en cuanto pueden aportar importantes elementos a sus estrategias publicitarias y de atención al cliente.

\section{Metodología}

La investigación tuvo un enfoque mixto trabajando dos etapas: cualitativa y cuantitativa. La población objetivo fueron los estudiantes universitarios de 20 a 27 años, de las universidades privadas de la ciudad de Santiago de Cali. En la primera etapa se realizó un grupo focal en el que participaron 8 personas y en la etapa cuantitativa se aplicó un cuestionario a una muestra de 80 personas, que corresponde una confianza del $90 \%$ y a un error del $10 \%$. El trabajo de campo se desarrolló en el mes de mayo de 2015.

El tipo de análisis realizado en la etapa cualitativa corresponde al interpretativo que busca comprender e interpretar, las percepciones del segmento en estudio, acerca de los sex-shop y la experiencia de compra de los juguetes sexuales. En la etapa cuantitativa se desarrolló un análisis a partir de la estadística descriptiva para conocer las tendencias de compra del sector en el segmento objetivo. Se muestra a continuación los resultados en donde se muestran los hallazgos desde el enfoque cualitativo y cuantitativo.

\section{Desarrollo}

En las últimas décadas la sexualidad en occidente se ha desligado poco a poco de la reproducción y toma más la forma de placer individual. Este cambio se ha visto motivado en parte, por el papel decisivo de la internet para manifestar el sexo de forma más abierta, pues se 
puede encontrar todo tipo de fantasías, contactos y demás contenidos sin censura; a esto se le añade la posibilidad de tener sexo virtual o cibersexo, en el cual no hay contenido físico, pero dos o más personas buscan placer sexual mediante este medio, pudiendo algunas veces terminar en citas o encuentros reales (Escalante et al, 2008). El concepto de sexo virtual es importante, pues el uso de juguetes sexuales, lubricantes y demás artículos adquiridos en sexshop pueden estar involucrados dentro de estas dinámicas, convirtiéndose en un factor importante a la hora de evaluar la compra.

Lo dicho anteriormente se ve reflejado en los resultados de la investigación. Desde el focus group, los participantes opinaron que el sexo es cada vez un tema más abierto que poco a poco deja de ser tabú con el cambio de generaciones, mientras que en la parte cuantitativa el $79 \%$ dijo sentirse cómodo al hablar de temas sexuales, representando en hombres un $91 \%$ de la muestra $\mathrm{y}$ en mujeres un $71 \%$.

\section{Percepción de los sexshops}

Mackenna (2013), habla de lo importante de la privacidad del cliente, por lo que estos sitios se aislan de su entorno con el fin de garantizar privacidad; esto se acompaña de iluminación tenue con luces de neón, creando un ambiente no del todo intimo, pero tampoco público sino como un espacio marginal entre ambos, teniendo en cuenta siempre un factor que lo diferencie de otros sex shop debido a que por lo general estos locales se juntan en zonas donde los clientes tienen una sensación de complicidad en la que "todos están en lo mismo", creando también una atmósfera de frontera, lo que comúnmente se llama en antropología un "no lugar". Es por todos estos aspectos, que los sex shop deben en parte su conocimiento al voz a voz.

Respaldado por los resultados, todos los participantes del focus group dicen saber de la existencia de los sexshops y de su ubicación en la ciudad, afirmando conocerlos por internet, redes sociales y amigos, además de volantes en la calle y los locales a plena vista por distintos sectores de la ciudad, resaltando este hecho que hace unos años era inpensable. En la parte cuantitativa, el $97 \%$ (100\% de los hombres y 95\% de las mujeres) dice haber escuchado sobre sexshops y el 94\% (100\% hombres y $90 \%$ mujeres) reconoce sus zonas habituales dentro de la ciudad.

En cuanto a la disposición del lugar, se encuentra una dualidad entre el humor y el placer, pues por un lado están los productos para fiestas con tema sexual, pero al mismo tiempo están los juguetes, lubricantes y demás productos relacionados al placer que, aunque generalmente tienen una percepción seria, se pueden tomar para bromas como un regalo a un amigo o como algo humorístico e infantil por las decoraciones de algunos, con dibujos animados o hello kitty, así como los nombres parodia y sugestivos de las películas pornográficas. Esta dualidad también aparece en los vendedores, que responden de forma natural y un poco picante y confidente. A través de esta dualidad, los productos se liberan de tensiones existentes, haciendo más fácil la compra.

Tabla 1. Percepción ingresar a un Sexshop

\begin{tabular}{llll}
\hline $\begin{array}{l}\text { Qué tan cómodo } \\
\text { creen es entrar } \\
\text { a un sexshop }\end{array}$ & $\begin{array}{l}\text { Femeni } \\
\text { no }\end{array}$ & $\begin{array}{l}\text { Mascul } \\
\text { ino }\end{array}$ & $\begin{array}{l}\text { Total } \\
\text { general }\end{array}$ \\
\hline $\begin{array}{l}\text { Ni cómodo ni } \\
\text { incómodo }\end{array}$ & $40 \%$ & $56 \%$ & $46 \%$ \\
$\begin{array}{l}\text { Cómodo } \\
\text { Incómodo }\end{array}$ & $25 \%$ & $22 \%$ & $24 \%$ \\
$\begin{array}{l}\text { Muy incómodo } \\
\text { Muy Cómodo }\end{array}$ & $10 \%$ & $6 \%$ & $9 \%$ \\
Total general & $\mathbf{1 0 0 \%}$ & $\mathbf{1 0 0 \%}$ & $\mathbf{1 0 0 \%}$ \\
\hline
\end{tabular}

Fuente: Elaboración propia.

En la etapa cuantitativa, los participantes expresaron la vergüenza que sentirían de entrar a uno de estos locales, mientras que de los encuestados un 24\% (22\% hombres y $25 \%$ mujeres) dice sería una experiencia cómoda y un $46 \%$ (40\% mujeres y $56 \%$ hombres) dice que no sería ni cómoda ni incómoda. En cuanto a la ambientación del lugar, se dio mucha importancia al servicio prestado al cliente, la 
información suministrada por el personal y la visibilidad de los productos, obteniendo cada una una calificación media de 4.6, 4.5 y 4.4 sobre 5, respectivamente. Los resultados se observan en la tabla 1.

Dentro de la tienda, los vendedores dejan espacio al cliente y no interactúan con este a menos que él lo haga primero, dando espacio libre, sin presiones para que el cliente observe y deje volar su imaginación. Cuando se les hace una pregunta responden de forma clara, natural y sin inhibiciones, haciendo siempre alusión al conocimiento del tema y de los productos, teniendo una relación cercana y simétrica diciendo que ellos también los han usado $\mathrm{y}$ recomendando alguno en específico. Los vendedores se convierten en guías dentro del sexshop.

Tabla 2. Experiencia lugar de compra

\begin{tabular}{|c|c|c|}
\hline Género & $\begin{array}{lr}\text { Es } & \text { más } \\
\text { importante } & \text { la } \\
\text { privacidad } & \text { de } \\
\text { comprar } & \text { en } \\
\text { internet } & \\
\end{array}$ & $\begin{array}{lr}\text { Es } & \text { más } \\
\text { importante } & \text { la } \\
\text { experiencia } & \text { de } \\
\text { comprar } & \text { en } \\
\text { punto de venta }\end{array}$ \\
\hline Mujeres & $25 \%$ & $75 \%$ \\
\hline Hombres & $31 \%$ & $69 \%$ \\
\hline General & $28 \%$ & $73 \%$ \\
\hline
\end{tabular}

Fuente: Elaboración propia.

Esto es exactamente lo que opinan los participantes del focus group cuando dicen esperar atención por parte del encargado, en una relación de complicidad donde se da una comunicación de tipo "amistosa", pues con sus amigos se sienten cómodos al hablar de temas de sexo. Esto va acompañado de la gran improtancia dada a la atención al cliente información entregada por el personal del sexshop, junto con un $73 \%$ de los encuestados (69\% hombres y 75\% mujeres) dicen preferir comprar artículos sexuales en un punto físico por la atención y experiencia de compra, que la privacidad de hacerlo por una página web. Ver tabla 2.
En cuanto a los juguetes sexuales, el 100\% de los encuestados dice haber escuchado hablar de estos y el $88 \%$ ( $88 \%$ hombres y $88 \%$ mujeres) considera normal su uso en personas solteras, considerando que lo hacen por buscar placer cuando no hay pareja (80\%), porque son innovadores $(33 \%)$, por aburrimiento (20\%) y porque son inseguros $(3 \%)$, los resultados se aprecian en la tabla 3.

Tabla 3. Uso de juguetes sexuales en personas solteras

\begin{tabular}{lrlll}
$\begin{array}{l}\text { Los solteros usan } \\
\text { juguetes } \\
\text { porque }\end{array}$ & $\begin{array}{l}\text { Fem } \\
\text { sexuales }\end{array}$ & $\begin{array}{l}\text { Mascul } \\
\text { ino }\end{array}$ & Total \\
\hline $\begin{array}{l}\text { Buscan } \\
\text { cuando no existe } \\
\text { pareja }\end{array}$ & $\begin{array}{r}\text { placer } \\
\text { ind }\end{array}$ & $83 \%$ & $75 \%$ & $\mathbf{8 0 \%}$ \\
$\begin{array}{l}\text { Son innovadores } \\
\text { Aburrimiento }\end{array}$ & $33 \%$ & $31 \%$ & $\mathbf{3 3 \%}$ \\
Son inseguros & $23 \%$ & $16 \%$ & $\mathbf{2 0 \%}$ \\
\hline
\end{tabular}

Fuente: Elaboración propia.

\section{Percepción del uso de juguetes sexuales en solteros}

En cuanto al uso por género, se le asocia más a las mujeres, especialmente con aquellos juguetes de forma fálica, como lo son vibradores (96\%) y dildos $(71 \%)$, dejando también un lugar significativo para la tanga vibradora (40\%). Mientras tanto a los hombres, solo se les asocia significativamente con las muñecas inflables (79\%).

En la mayoría de los casos, las mujeres tienen una mayor percepción de uso de juguetes sexuales para su mismo género, un ejemplo es que el $50 \%$ de las mujeres creen que las mujeres usan tangas vibradoras, frente a un $25 \%$ de los hombres que lo cree. En el caso masculino este patrón se repite, pero de forma más sorprendente, pues se ve incluso en el uso de juguetes usados para la penetración, como en el caso de los vibradores en el que el $15 \%$ de las mujeres creen que los hombres lo usan, frente a un $31 \%$ de los hombres que lo cree; igualmente 
sucede con los dildos en el que $21 \%$ de las mujeres creen que los hombres lo usan, mientras que el $41 \%$ de ellos opina esto mismo.

Estos resultados reflejan la historia de los juguetes sexuales y su desarrollo dentro de la sociedad, pues se ha encontrado que los dildos se usan desde al menos el siglo III en Grecia y los dildos de goma que ahora se conocen datan del siglo XIX cuando eran usados en tratamientos médicos con la aprobación de sus pacientes para controlar a las mujeres y curar enfermedades como la histeria. En los años 30 ya algunas revistas para mujeres promocionaban los vibradores sin que estos apareciesen ni tuviesen mensajes sexuales. Estos empezaron a aparecer en publicidad después de la revolución sexual (Fash \& Swank, 2013).

Esto también se refleja en concepciones sociales sobre las mujeres, como por ejemplo que es muy difícil llegar al orgasmo o que deben complacer sexualmente al hombre sin importar lo que ellas quieran o queden satisfechas y que mediante el uso de juguetes sexuales las mujeres pueden ser proporcionadas de un estado de liberación al hacer lo que no se espera de ellas y desligarse de lo que siempre se ha considerado "correctamente sexual", convirténdose pues en un símbolo del cuestionamiento y paulatina destrucción de las prácticas sexuales naturalizadas. De esta forma, por ejemplo un dildo no suple una falta, sino que desplaza la concepción del sexo reproductivo al placentero, convirtiéndose en una clase de prótesis para la mujer (Mora, 2010).

Debido a esta concepción de empoderamiento femenino por el uso de juguetes sexuales, encontramos claras referencias en revistas populares como Cosmopolitan o series como Sex and the city o en el caso nacional, la serie online Susana y Elvira.

\section{Percepción del uso de juguetes sexuales en pareja}

En cuanto al uso de juguetes sexuales por parejas, el $78 \%$ de los encuestados lo considera normal, mientras que el $18 \%$ piensa que esto depende del tiempo que lleve junta la pareja.

Igualmente, en el focus group, cuando se preguntó sobre los consumidores de juguetes sexuales, la atención se centró en parejas, teniendo en cuenta conceptos como la complicidad, comodidad, libertad y conocimiento mutuo. Cuando se preguntó el por qué de esto, afirmaron haber visto varias parejas entrar a distintos sexshops, además de opinar que la mayoría de los juguetes sexuales están diseñados para el disfrute en pareja debido a la monotonía que los motiva a buscar nuevas experiencias para "revivir la pasión", mientras que uno de los participantes relacionó el uso de juguetes sexuales en pareja como inseguridad por parte del hombre para cumplir "su labor" y otro opinó que son personas innovadoras; afirmación que es apoyada en la encuesta, pues el $70 \%$ considera que las parejas que hacen uso de juguetes sexuales son innovadores, el $71 \%$ considera que existe mayor confianza y esto permite discutir el tema, mientras que el $41 \%$ dice que los usan porque la relación se vuelve monótona y un $4 \%$ afirma que uno de sus integrantes es inseguro. Los resultados se muestran en la tabla 4.

En cuanto a los juguetes que usan las parejas, los encuestados apoyan las conclusiones del focus group; "las parejas usan más juguetes sexuales que los solteros" y "los juguetes sexuales están diseñados más para parejas que para solteros" pues todas las categorías obtuvieron un pocentaje significativo en los resultados.

Socialmente se acepta que los juguetes sexuales pueden añadir diversidad a la relación de pareja y ayuda a personas con traumas sexuales y de autoimagen. Los dildos y demás juguetes sexuales también pueden ser de uso masculino, pudiendo incluso experimentar penetraciones por parte de sus parejas (heterosexuales) trascendiendo los estereotipos de los roles sexuales (Fash \& Swank, 2013).

Tabla 4. Motivo de uso de juguetes sexuales 


\begin{tabular}{|c|c|c|c|}
\hline $\begin{array}{l}\text { Los solteros } \\
\text { usan juguetes } \\
\text { sexuales } \\
\text { porque }\end{array}$ & $\begin{array}{l}\text { Femenin } \\
\text { o }\end{array}$ & $\begin{array}{l}\text { Masculin } \\
\text { o }\end{array}$ & $\begin{array}{l}\text { Tota } \\
\text { l }\end{array}$ \\
\hline $\begin{array}{l}\text { Existe mayor } \\
\text { confianza para } \\
\text { explorar } \\
\text { nuevos gustos }\end{array}$ & $73 \%$ & $69 \%$ & $71 \%$ \\
\hline $\begin{array}{l}\text { Son } \\
\text { innovadores }\end{array}$ & $75 \%$ & $63 \%$ & $70 \%$ \\
\hline $\begin{array}{l}\text { La relación es } \\
\text { monótona }\end{array}$ & $35 \%$ & $50 \%$ & $41 \%$ \\
\hline \begin{tabular}{lc}
\multicolumn{3}{l}{ Inseguridad } \\
por parte & de \\
uno de & los \\
integrantes &
\end{tabular} & $6 \%$ & $0 \%$ & $4 \%$ \\
\hline
\end{tabular}

Fuente: Elaboración propia.

Los juguetes sexuales también pueden ser vistos como una continuación de los estereotipos sexuales y un beneficio capitalista que se disfraza en uno social (el empoderamiento de la mujer). Un ejemplo de los estereotipos es como los empaques de los vibradores tienen mujeres en poses sugestivas, sacadas del típico estereotipo de actriz porno, porque los fabricantes esperan atraer así al público masculino que le compra los juguetes a "su" mujer, intentando reflejarla en el empaque y detrás de eso cumplir sus propias fantasías pornográficas o en el caso de los dildos negros que aluden al racismo y los estereotipos, pues todos los de color negro son gigantes, produciendo terror y deseo, soportando la idea de que los hombres negros son agresivos sexualmente (Fash \& Swank, 2013).

\section{Percepción del mercado}

Esto nos lleva a hablar de la percepción del mercado de los juguetes sexuales y los sexshops, respecto a la cual, los participantes del grupo focal opinan que es un negocio lucrativo y en aumento pues cada vez hay más sexshop en la ciudad y consideran importante la compra en este establecimiento, pues tienen derecho a asesoría personalizada de un conocedor del tema, además de mayor confianza en el producto, pues por internet puede llegar roto, abierto, usado, etc. Así mismo, consideran que la publicidad utilizada es poco llamativa $\mathrm{y}$ efectiva, creyendo que se podría mejorar si rompe aún más el tabú y se marginan los miedos, comunicando los servicios que presta cada producto, creando así nuevas necesidades ofreciendo experiencias que generan placer.

En cuanto a los encuestados, el $46 \%$ afirma haber visto publicidad de sexshops, de los cuales el $64 \%$ lo ha hecho a través de volantes, el $22 \%$ por redes sociales $(71 \%$ en facebook y $29 \%$ en instagram), el $11 \%$ por páginas de ventas de todo tipo y el $3 \%$ por radio. De estos mismos, el $46 \%$ la considera regular, el $24 \%$ buena, $22 \%$ mala y $8 \%$ muy mala.

El $45 \%$ de los encuestados ha visto publicidad de juguetes sexuales, de los cuales el $56 \%$ los ha visto en páginas web de ventas, el $44 \%$ en redes sociales (50\% facebook y $50 \%$ instagram), el $33 \%$ en volantes y el $3 \%$ en radio, siendo considerada buena por un $39 \%$, regular por otro $39 \%$ y mala por el $22 \%$.

Una ventaja para aprovechar en el mercado, fue encontrada durante una investigación (Mora, 2010) donde se descubrió que las entrevistadas se sentían como un objeto usado para complacer sexualmente al hombre o para llevar a cabo la reproducción, situación que generaba malestar emocional, autoprohibiciones y culpas en relación a sus deseos sexuales, junto con curiosidad y deseo de explorar e ir en contra de la normativa sexual dominante. A partir de esto se identificaron 5 etapas para el uso de juguetes sexuales que se podrían comparar con las etapas o fases de compra:

Motivación: curiosidad y deseo de innovar en sus prácticas sexuales (Reconocimiento de la necesidad).

Autojuzgamiento: se preguntan si su comportamiento es normal y temen ser juzgadas. (Búsqueda de información).

Tomar el riesgo: cuando usan el juguete sexual. Pueden comprarlo ellas mismas y usarlo 
sola o con su pareja. Evaluación de alternativas y decisión de compra).

Búsqueda de legitimidad: Tiene que creer en su nueva propuesta de práctica sexual buscando información en internet, libros, películas pornográficas, revistas y personas cercanas, las cuales son las más importantes, pues si la juzgan de forma favorable el sentimiento de culpa disminuye y empieza a ver esta práctica como normal; sin embargo, si la juzgan la mujer se quedará callada y no dejará de dudar de lo malo de esta nueva práctica. (Búsqueda de la información o rectificación de ésta).

Resolución positiva o negativa: es positiva si la mujer ya no tiene dudas, temores o culpas, se siente más libre y tiene mayor conciencia sobre su cuerpo y su sexualidad, siendo posible explorar aún más en su sexualidad. Es negativa cuando el juguete sexual se usa para el placer de su pareja y no suyo. Lo más normal es encontrar una resolución que se mueve entre ambos polos, usando estos satisfactoriamente consigo mismas y con su pareja, pero aún teniendo dudas menores sobre su comportamiento. Un ejemplo es pensar que si van a un sex shop se encontrarán a algún conocido. Comportamiento posterior a la compra)

\section{Conclusiones}

Para el grupo elegido, hablar sobre temas sexuales no representa un gran reto o tabú y por el contrario, en su mayoría se encuentran muy dispuestos a hablar y compartir sus pensamientos. Los sexshops y juguetes sexuales son bastante conocidos y no representan nada extraño o ajeno a su mundo, identificando estos sitios en lugares cercanos a sus casas o de paso camino a esta y aunque muestran interés, muchos de ellos se sienten cohibidos a entrar a este tipo de establecimientos por miedo a ser vistos o encontrar algún conocido dentro.

El conocimiento de los juguetes sexuales en muchas ocasiones se deriva de la experiencia adquirida hablando con amigos, leyendo revistas o libros y viendo películas y series de televisión. Muchos de estos productos, también tienen una deducción lógica o aparentemente lógica por su nombre, lo que hace pensar a los jóvenes que los conocen cuando realmente existen confusiones, tal es el caso de la funda para pene que es un juguete sexual diseñado para disfrute en pareja, pero muchos de los participantes en ele studio, por el hecho de contener la palabra "pene" lo enmarcaron dentro del consumo masculino, mismo caso con los anillos vibradores. En general, se podría sacar una gran idea de negocio e impulsar este tipo de tienda y consumo si se llegara al público con comunicación más asertiva y abierta.

Los juguetes sexuales son percibidos en su mayoría para uso en pareja y en caso de hacerse un uso como soltero, se relaciona más con las mujeres que con los hombres. En el caso de la percepción de uso de juguetes sexuales por parte de mujeres solteras, éstas tienen una percepción más alta de uso que los hombres, situación que se revierte en el caso de la percepción de uso de juguetes sexuales por parte de hombres solteros, donde ellos tienen una percepción de uso más alta que el de las mujeres. Tanto para parejas como para solteros, la mayor parte de este grupo coincide en que es una práctica normal que normalmente se relaciona a la innovación, la confianza y la búsqueda de placer bien sea personal o en pareja.

En cuanto al sector, podemos concluir que hay grandes oportunidades de mercado que están siendo desaprovechadas, al no incluir un buen uso del merchandising en el punto de venta, publicidad de los sexshops y juguetes sexuales, además del uso de frecuencias de horas donde las personas están más dispuestas a entrar. Como demuestran los resultados, los jóvenes buscan encontrar en el sexshop un guía y confidente que los aconseje y oriente, tema que también debe ser aprovechado junto con la promoción de juguetes sexuales "eco" para sacarle la cara buena a una desventaja del mercado sexual.

Por otra parte hay una gran oportunidad en la enseñanza a través de una comunicación eficiente de los distintos juguetes sexuales y sus usos, para disminuir el tabú y ser más exitosos y 
asertivos al marginar ciertos miedos, aprovechando el proceso de compra analógico desarrollado por Mora. Por lo que sería necesaria toda la implantación de estos detalles olvidados por el mercado para crear un negocio exitoso aumentando el lucro al incentivar una necesidad que cada vez más se busca suplir aún por encima del tabú o las barreras que impone la sociedad.

\section{Referencias}

Caraballo, G. (2010, 31 de mayo). "Los 'sex shops' en Bogotá venden más de cinco millones de dólares al año", sitio web de Portafolio [en línea], disponible en: http://www.portafolio.co/archivo/documento/C MS-7733139

Escalante, L., Cerrón, R., Salazar, A., \& Mezones, E. (2008). "Descripción de la conducta sexual en adultos jóvenes limeños". Revista Horizonte Médico , 8 (1).

Fash, B., \& Swank, E. (2013). "Adventures with the "Plastic Man": Sex toys, compulsory heterosexuality, and the politics of women's sexual pleasure". Sexuality \& Culture, 17, 666 685.

Finanzas Personales. "¿Cuánto cuesta ser Christian Grey?". Sitio web de Finanzas Personales, [en línea], disponible en: http://www.finanzaspersonales.com.co/consumo -inteligente/articulo/cuanto-cuesta-christiangrey/55523, [accesado el 22 de febrero de 2015]

Mackenna, B. (Octubre, 2013). "Consumo y normatividad: Prácticas de consumo asociadas a sexshops y tiendas de parafernalia cannábica en Santiago de Chile". XXIX Congreso de la Asociación Latinoamericana de Sociología. Santiago de Chile.

Mora, M. (2010). "Significados construidos en la práctica de usar juguetes sexuales por mujeres de clase media de la Región Metropolitana: Un Análisis desde la psicología comunitaria". Universidad de Chile, Facultad de Ciencias Humanas, Santiago de Chile.

Ramírez, L. P. (2011, 2 de septiembre). Sex Shop mueven 1 millón de dólares anuales. Mercado de Dinero. Recuperado de: http://www.mercadodedinero.com.co/Consumo/ 1959-sex-shop-mueven-1-millon-de-dolaresanuales.html

Sloan, K. (2014). The greening of sex toys. Herizons, 28 (1), 28 - 31.

Tabares, E.C. (2011, 25 de noviembre). Los sex shop, un mercado que se impone. Periódico La Tarde. Recuperado de: http://www.latarde.com/historico/51630-los-sexshop-un-mercado-que-se-impone 